\title{
Cuestionario CAVICAVENMI: calidad de vida en pacientes con cáncer de vejiga no músculo invasivo
}

\section{CAVICAVENMI: Quality of Life Survey in Non-muscle- invasive Bladder Cancer Patients}

\author{
Irache Abáigar-Pedraza ${ }^{1}$ Julián Megías-Garrigós ${ }^{1}$ José Sánchez-Payá2 Sara Calvo Simal ${ }^{3}$
}

${ }^{1}$ Servicio de Urología, Hospital General Universitario de Alicante, Alicante, Spain

2 Servicio de Epidemiología y Medicina Preventiva, Hospital General Universitario de Alicante, Alicante, Spain

${ }^{3}$ Unidad de Investigación, Hospital Universitario de Burgos, Burgos, Spain
Address for correspondence Irache Abáigar Pedraza, Servicio de Urología, Hospital General Universitario de Alicante,

Bloque B, 7a Planta, Avda. Pintor Baeza, 12, 03010 Alicante, Spain (e-mail: iramedi@hotmail.com).

Urol Colomb 2019;28:161-168.

\section{Resumen}

\section{Palabras Clave}

- cáncer de vejiga

- cáncer de vejiga no músculo invasivo

- calidad de vida

- calidad de vida relacionada con la salud

- cuestionario

Abstract
Objetivo Evaluar la calidad de vida en pacientes diagnosticados de cáncer de vejiga no músculo invasivo a lo largo del estudio utilizando el cuestionario CAVICAVENMI. Material y Método Se incluyen 180 pacientes diagnosticados de TVNMI (febrero 2013 junio 2015). Todos rellenaron el cuestionario CAVICAVENMI en cuatro ocasiones: previo a la cirugía y la primera, segunda y tercera visita postintervención. Aquellos que reciben tratamiento adyuvante completan el cuestionario en dos ocasiones más: a mitad y final del ciclo de tratamiento. Estudiamos la variabilidad en la calidad de vida con una T-Student para datos apareados. Realizamos T - Student para muestras independientes entre el decremento porcentual (antes de la intervención frente a tercera visita postintervención) y determinadas características tumorales (tamaño, número de siembras, localización y CIS - carcinoma in situ). Realizamos un estudio de regresión múltiple para determinar valores de confusión entre las características tumorales.

Resultados Evidenciamos una mejora significativa en la calidad de vida a partir de la tercera visita postintervención en pacientes sin tratamiento adyuvante; y a partir del segundo momento postintervención en aquellos con tratamiento adyuvante. No existen diferencias significativas entre las características tumorales, pero observamos una mayor puntuación en presencia de las mismas. No encontramos factores de confusión.

Conclusiones La calidad de vida en pacientes TVNMI, presenta una mejoría a lo largo del estudio. Esos cambios son más tempranos en los apartados de percepción de enfermedad, autoestima y estado emocional. Aquellos pacientes con presencia de las características tumorales estudiadas, presentan una peor calidad de vida, aunque no es estadísticamente significativo.

Purpose To evaluate the quality of life of patients diagnosed with NMIBC throughout the time of the study, using the CAVICAVENMI questionnaire. received

May 17, 2017

accepted

October 3, 2017
DOI https://doi.org/

10.1055/s-0038-1656516.

ISSN 0120-789X.

eISSN 2027-0119.
Copyright $($ C 2019, Sociedad Colombiana License terms de Urología. Publicado por Thieme Revinter Publicações Ltda., Rio de Janeiro, Brazil. Todos los derechos reservados.

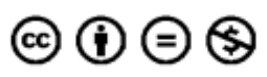




\section{Keywords}

- bladder cancer

- non-muscle-invasive bladder cancer

- quality of life

- health quality

- questionnaire and questionnaire design
Material and Methods 180 patients diagnosed with NMIBC were included (February 2013 - June 2015) and completed the questionnaire on four occasions: prior to surgery, and in the first, second and third visits post-surgery. Patients on adjuvant treatment also completed it at the middle and final treatment cycle. Student's $t$-test was used to study the variability of the paired data in the quality of life questionnaire. An analysis (Student's-t Test) was made between the percentage decrease (before the intervention compared with the third post-surgery visit) and certain tumor characteristics (size, seeding number, location and carcinoma in situ) using. Multiple regression analysis was performed to determine confundant elements between tumor characteristics

Result A statistically significant improvement in the quality of life was observed from the third post-intervention visit in patients that did not receive instillations, and in patients that received instillations, this significant improvement appeared from the second post-intervention visit. No statistically significant differences were found in the tumor characteristics, but a higher score was observed in the presence of these. We do not find confundant elements.

Conclusions The quality of life in NMIBC patients showed an improvement throughout the study. These changes are seen earlier in the perception of the disease, in self-esteem and emotional state. Those patients with the presence of the tumor characteristics studied had a poorer, but not statistically significant, quality of life.

\section{Introducción}

En 1994 el grupo WHOQOL de la OMS define la calidad de vida como: "la percepción que un individuo tiene de su lugar en la existencia, en el contexto de la cultura y del sistema de valores en los que vive y en relación con sus objetivos, sus expectativas, sus normas, sus inquietudes". 1,2 Desde entonces, la valoración de la calidad de vida y, específicamente, "la calidad relacionada con la salud de la vida" (CVRS), ha ganado terreno en el campo de la investigación, convirtiéndose en una de las herramientas más importantes en la práctica diaria asistencial. Hemos observado un aumento exponencial en el uso de herramientas que evalúan el impacto en la CVRS, con el fin de valorar los beneficios humanos y costes económicos de los diferentes campos que acoge el sistema de salud, desde la presencia de enfermedades hasta su tratamiento médico y/o quirúrgico. $^{3}$

Dentro de la urología, una patología tumoral con una incidencia no desdeñable en nuestro país, es el TVNMI, siendo el segundo tumor más frecuente del aparato urinario después del cáncer de próstata. ${ }^{4}$ En nuestro día a día evidenciamos que dicha patología causa gran repercusión en la calidad de vida. Hasta finales de 2014, las herramientas utilizadas para medir ese impacto no estaban adecuadamente validadas ni adaptadas a los diferentes idiomas por lo que los resultados obtenidos no podían ser extrapolados ni comparados. A partir de esa fecha, se publicaron la validación del EORTC QLQ-NMIBC24 ${ }^{5}$ y la adaptación al español ${ }^{6}$ del cuestionario Bladder Cancer Index $(\mathrm{BCI})^{7}$
En 2012 comenzamos el proceso de elaboración y validación de un cuestionario (CAVICAVENMI) cuyos resultados han sido publicados. ${ }^{8}$

El objetivo de este estudio es determinar la variabilidad de la calidad de vida utilizando el CAVICAVENMI a lo largo del tiempo de estudio en pacientes con TVNMI.

\section{Material y Métodos}

Se realiza un estudio prospectivo para evaluar la calidad de vida a lo largo del tiempo en pacientes diagnosticados de TVNMI utilizando el cuestionario CAVICAVENMI. Entre febrero 2013 y junio 2015, se incluyeron de manera consecutiva, previa solicitud de consentimiento informado, pacientes diagnosticados con TVNMI. A todos se les administró el cuestionario CAVICAVENMI. El número inicial de pacientes fue de 205. Durante la fase de diseño del cuestionario, ${ }^{8}$ no se realizó un proceso de estimación de la muestra porque como describimos en el artículo previo de validación y fiabilidad del cuestionario, no encontramos datos en la literatura científica para realizar dicha estimación de manera formal. ${ }^{8}$ Por lo que, dada la frecuencia de esa patología, se decidió seleccionar un número amplio de pacientes de manera consecutiva. Fueron excluidos los pacientes que presentaron progresión de la enfermedad o recidiva, respondieron a menos de un $50 \%$ de las preguntas o abandonan el tratamiento con Mitomicina $\mathrm{C}$ (MMC) o bacilo de Calmette-Guérin (BCG). El número final de pacientes incluidos fue de 180 ( - Fig. 1). En el momento de la inclusión y tras la cirugía, se recogieron las características 


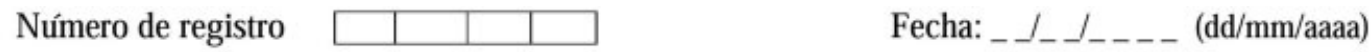

Cuestionario sobre la calidad de vida en pacientes con cáncer de vejiga no músculo invasivo(CAVICVENMI) Marque la casilla con una $X$

EN CUANTO A LA ENFERMEDAD (E)

Nada $\quad$ Poco Algo Mucho Muchísimo

\begin{tabular}{l|l|l|l|l}
$(0)$ & $(1)$ & $(2)$ & $(3)$ & $(4)$
\end{tabular}

1. ¿Se le escapa la orina?

2. ¿Presenta dolor al orinar?

3. ¿Está preocupado por la aparición de sangre en la orina?

4. ¿Le produce ansiedad pensar en la cistoscopia?

5. ¿Son dolorosas las cistoscopias?

\begin{tabular}{|c|c|c|c|c|c|}
\hline AUTOESTIMA Y ESTADO EMOCIONAL (A) & $\begin{array}{c}\text { Nada } \\
(0)\end{array}$ & $\begin{array}{l}\text { Poco } \\
(1)\end{array}$ & $\begin{array}{l}\text { Algo } \\
(2)\end{array}$ & $\begin{array}{c}\text { Mucho } \\
(3)\end{array}$ & $\begin{array}{l}\text { Muchísimo } \\
\text { (4) }\end{array}$ \\
\hline \multicolumn{6}{|l|}{ 1. ¿Se siente triste? } \\
\hline \multicolumn{6}{|l|}{ 2. ¿Está más irritable? } \\
\hline \multicolumn{6}{|l|}{ 3. ¿Se encuentra más distanciado de su familia? } \\
\hline \multicolumn{6}{|l|}{ 4. ¿Le preocupa que su enfermedad emperore? } \\
\hline \multicolumn{6}{|l|}{ 5. ¿Piensa que puede morir antes? } \\
\hline VIDA LABORAL (L) & $\begin{array}{c}\text { Nada } \\
(0)\end{array}$ & $\begin{array}{l}\text { Poco } \\
\text { (1) }\end{array}$ & $\begin{array}{l}\text { Algo } \\
(2)\end{array}$ & $\begin{array}{c}\text { Mucho } \\
(3)\end{array}$ & $\underset{\text { (4) }}{\text { Muchísimo }}$ \\
\hline \multicolumn{6}{|l|}{$\begin{array}{l}\text { 1. ¿Se ve incapacitado para realizar su trabajo, incluidas las tareas del } \\
\text { hogar? }\end{array}$} \\
\hline $\begin{array}{l}\text { 2. ¿Ha disminuido el tiempo que dedica a su trabajo, incluyendo las } \\
\text { tareas del hogar? }\end{array}$ & & & & & \\
\hline
\end{tabular}

\begin{tabular}{|c|c|c|c|c|c|}
\hline VIDA DIARIA (V) & $\begin{array}{c}\text { Nada } \\
(0)\end{array}$ & $\begin{array}{c}\text { Poco } \\
\text { (1) }\end{array}$ & $\begin{array}{l}\text { Algo } \\
(2)\end{array}$ & $\begin{array}{l}\text { Mucho } \\
(3)\end{array}$ & $\begin{array}{l}\text { Muchísimo } \\
\text { (4) }\end{array}$ \\
\hline 1. ¿Ha tenido que cambiar sus planes de futuro? & & & & & \\
\hline 2. ¿Se han afectado las actividades de su vida diaria? & & & & & \\
\hline $\begin{array}{l}\text { 3. Debido a su enfremedad ¿ha disminuido el tiempo que dedica a su } \\
\text { actividades lúdicas? }\end{array}$ & & & & & \\
\hline $\begin{array}{l}\text { 4. Debido a la enfermedad, ¿ha cambiado el tiempo que dedica a sus } \\
\text { amistades? }\end{array}$ & & & & & \\
\hline $\begin{array}{l}\text { 5. Si realiza ejercicio (caminar, correr, nadar...) ¿ha disminuido el } \\
\text { tiempo que le dedica? }\end{array}$ & & & & & \\
\hline
\end{tabular}

\begin{tabular}{|c|c|c|c|c|c|}
\hline VIDA SEXUAL (S) & $\begin{array}{c}\text { Nada } \\
(0)\end{array}$ & $\begin{array}{c}\text { Poco } \\
\text { (1) }\end{array}$ & $\begin{array}{c}\text { Algo } \\
(2)\end{array}$ & $\begin{array}{c}\text { Mucho } \\
(3)\end{array}$ & $\underset{\text { (4) }}{\text { Muchísim }}$ \\
\hline 1. ¿Se siente sexualmente rechazado por su pareja? & & & & & \\
\hline 2. ¿Ha diminuido su apetito sexual? & & & & & \\
\hline 3. ¿Ha disminuido el número de relaciones sexuales con su pareja? & & & & & \\
\hline $\begin{array}{l}\text { 4. ¿Piensa que su enfermedad puede transmitirse con las relaciones } \\
\text { sexuales? }\end{array}$ & & & & & \\
\hline
\end{tabular}

Fig. 1 Cuestionario sobre la calidad de vida en pacientes con cáncer de vejiga no músculo invasivo (CAVICVENMI).

demográficas y una serie de características tumorales que conocemos que repercuten negativamente en la calidad de vida: tumor mayor a $3 \mathrm{~cm}$, tres o más siembras, localización en cúpula o trígono vesical y CIS positivo ( - Tabla $\mathbf{1}$ ).
El cuestionario se cumplimentó en varias ocasiones: la tarde previa a la cirugía, la primera visita después de la intervención (al cabo de un mes), la segunda visita después de la intervención (al tercer mes) y la tercera visita después 
Tabla 1 Características demográficas y tumorales $(n=180)$

\begin{tabular}{|l|l|}
\hline Edad (media \pm DS) & $70,3 \pm 10,28$ \\
\hline Sexo (frec, \%) & $(146) 81,1$ \\
\hline Varón & $(34) 18,9$ \\
\hline Mujer & $(64) 35,6$ \\
\hline Tamaño tumoral (n, \%) & $(116) 64,4$ \\
\hline$\geq 3 \mathrm{~cm}$ & $(52) 28,9$ \\
\hline$<3 \mathrm{~cm}$ & $(128) 71,1$ \\
\hline Número de siembras (n, \%) & \\
\hline$\geq 3$ & $(73) 41,1$ \\
\hline$<3$ & $(107) 58,9$ \\
\hline Localización (n, \%) & \\
\hline Cúpula y/o trígono & $(13) 7,2$ \\
\hline Otro & $(157) 92,8$ \\
\hline Carcinoma in situ (n, \%) & \\
\hline Positivo & \\
\hline Negativo &
\end{tabular}

de la intervención (al noveno mes si TVNMI-Ta o T1 G1 o al sexto mes si TVNMI-T1 G2 y G3 y aquellos con CIS). Si precisaban de tratamiento adyuvante con instilaciones con Mitomicina C MMC o BCG también cumplimentaban el cuestionario a la mitad y al final del ciclo de inducción.

Es una herramienta autoadministrada de 21 preguntas, con cinco respuestas distribuidas según una escala tipo Likert (Nada / Poco / Algo / Mucho / Muchísimo) a mayor puntuación obtenida una peor calidad de vida. La puntuación de cada pregunta va de 0 que corresponde a "Nada"; a 4, que corresponde a "Muchísimo." El rango de puntuación del cuestionario va de 0 a 84; siendo 0 la mejor calidad de vida posible y 84 la peor (-Fig. 2). Cuando el paciente recibe instilaciones de BCG o MMC se añade una nueva dimensión, denominada "tratamiento". En ese caso la puntuación va de 0 a 104 (-Fig. 3).

Cumplimentados los cuestionarios, se compararon con una $\mathrm{T}$ Student para datos apareados las medias de las puntuaciones obtenidas, en cada apartado y en el sumatorio global, antes de la intervención con los diferentes momentos a lo largo del estudio. (-Tabla 2 pacientes sin instilaciones de BCG o MMC y - Tabla 3 pacientes con tratamiento de BCG o MMC).

Posteriormente se calculó el decremento porcentual del cuestionario. Para ello se halló la diferencia porcentual entre las puntuaciones de cada dimensión y global del momento de la tercera visita postintervención con respecto al momento inicial antes de la cirugía. Después, se analizó la relación entre el decremento porcentual y las características tumorales descritas previamente mediante el test de la $\mathrm{T}$ de Student. (-Tabla 4). Además, se realizó un estudio univariante ( $\mathrm{T}$ student) entre las características tumorales y la calidad de vida en la terccera visita postintervención; para aquellas variables que obtuvieron un resultado significativo, se llevó a cabo un estudio de regresión lineal múltiple (-Tabla 5). Para el análisis estadístico, el nivel de significación estadística utilizado fue $\mathrm{p} \leq 0,05 \mathrm{y}$ para realizar los cálculos se utilizó el programa IBM-SPSS Versión 21.0.

\section{Resultados}

La media de edad de pacientes es de más de 70,3 $\pm 10,28$ años, en su mayoría hombres (81\%). Respecto a sus características tumorales, un $35,6 \%$ de tumores mayores a $3 \mathrm{~cm}, 28,9 \%$ más de tres siembras, 41,3\% localizados en trígono o cúpula vesical y CIS positivos en el 7,2\% de los casos (-Tabla $\mathbf{1}$ ).

De los 180 pacientes, 143 pacientes fueron sometidos a RTU y a 37 se les administró terapia endovesical adyuvante con BCG o MMC de acuerdo al protocolo de las guías clínicas para TVNMI.

No evidenciamos ningún cambio estadísticamente significativo en los resultados obtenidos antes de la intervención frente a la primera visita postintervención, excepto para el apartado de autoestima y estado emocional, donde los pacientes presentan una leve mejoría de la calidad de vida respecto al estado preoperatorio (p: 0,017). En la comparación entre el momento antes de la intervención con respecto a la segunda visita postintervención, encontramos diferencias estadísticamente significativas en el apartado autoestima y estado emocional (p: 0,01), vida diaria ( $p: 0,01$ ) $\mathrm{y}$ vida laboral $(\mathrm{p} \leq 0,001)$. Esas diferencias estadísticamente significativas, no se evidencian en la puntuación global. Sin embargo, la comparación con la tercera visita obtiene una

\section{Rellenar en caso de estar recibiendo tratamiento con BCG o MMC}

\begin{tabular}{|c|c|c|c|c|c|}
\hline TRATAMIENTO (T) & $\begin{array}{c}\text { Nunca } \\
(0)\end{array}$ & $\begin{array}{c}\text { Poco } \\
(1)\end{array}$ & $\begin{array}{c}\text { Algo } \\
\text { (2) }\end{array}$ & $\begin{array}{c}\text { Mucho( } \\
\text { 3) }\end{array}$ & $\begin{array}{c}\text { Muchísimo } \\
\text { (4) }\end{array}$ \\
\hline \multicolumn{6}{|c|}{ 1.¿Le produce ansiedad el pensar que tiene que recibir tratamiento? } \\
\hline \multicolumn{6}{|l|}{ 2.Tras la instilación ¿ha sentido dolor o molestias urinarias? } \\
\hline \multicolumn{6}{|l|}{ 3.¿El tratamiento recibido le ha condicionado su vida diaria? } \\
\hline \multicolumn{6}{|l|}{ 4. ¿Le preocupa que el tratamiento afecte a su vida sexual? } \\
\hline 5. ¿Repetiría el tratamiento si fuera necesario? & & & & & \\
\hline
\end{tabular}

Fig. 2 Rellenar en caso de estar recibiendo tratamiento con BCG o MMC. 


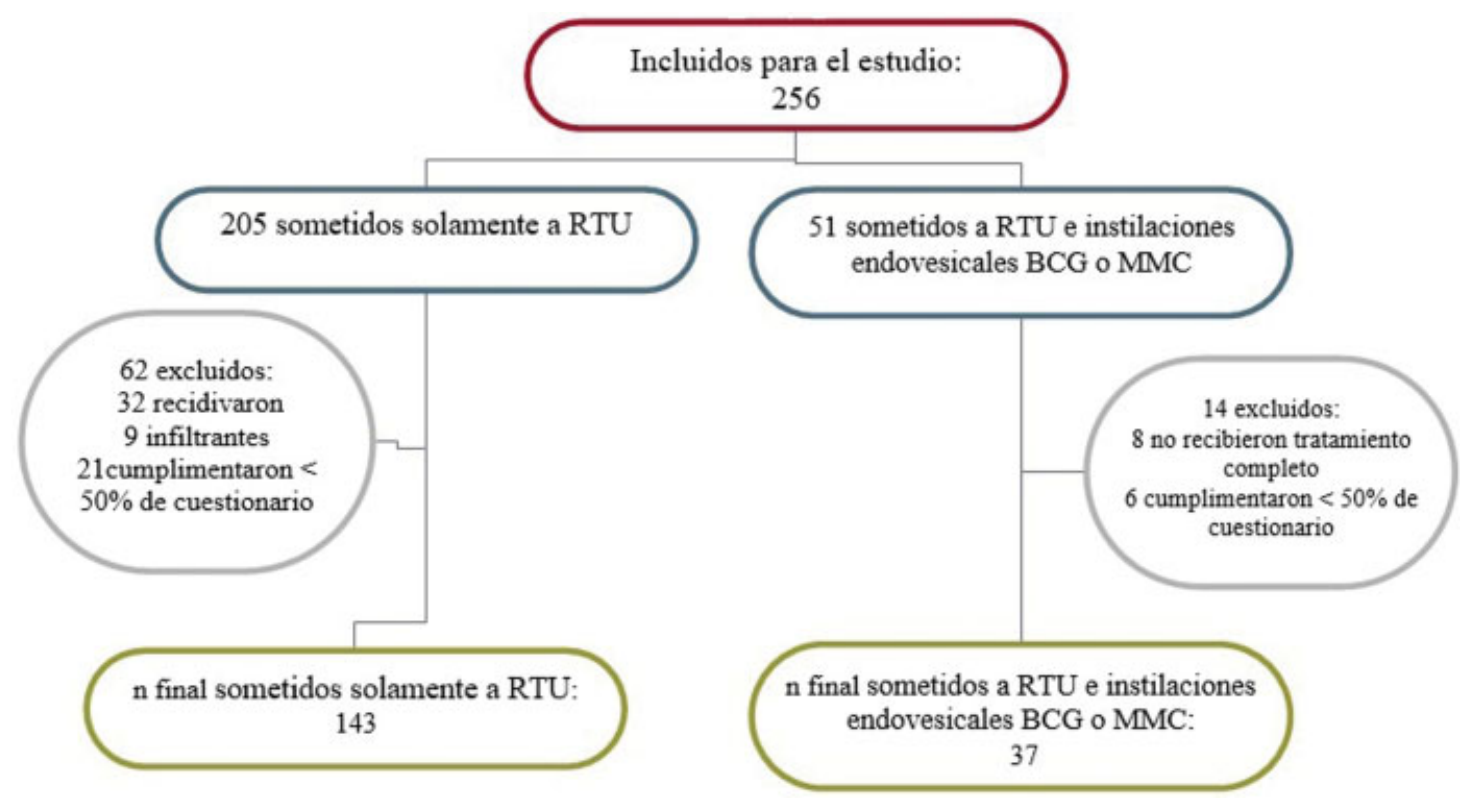

Fig. 3 Diagrama de flujo.

disminución de las puntuaciones en todas las dimensiones incluido el apartado global, encontrando en todos los casos diferencias estadísticamente significativas (-Tabla 2 ).

En los pacientes que reciben tratamiento endovesical adyuvante, evidenciamos un empeoramiento de la calidad de vida cuando se encuentran a mitad o final de ciclo de tratamiento en todos los apartados que en las dimensiones: "en cuanto a la enfermedad" y "autoestima y "estado emocional" no resulta significativo pero sí es estadísticamente significativo en la "vida laboral," "vida diaria" y "vida sexual", tanto a mitad como al final del ciclo. La puntuación global casi se duplica a mitad y final de ciclo con diferencias estadísticamente significativas con respecto a antes de la intervención, $44,5 \pm 13,7(<0,001)$ a mitad de ciclo y 41,0 $\pm 13,6(p<0,001)$ al final del ciclo.
Por otro lado, en ese grupo de pacientes, no observamos cambios estadísticamente significativos de mejora de la calidad de vida hasta la comparación del momento de antes de la intervención frente a la segunda visita postintervención que describe resultados estadísticamente significativos en todos los apartados incluso en el global al igual que ocurre en la comparación entre antes de la intervención y tercera visita postintervención ( - Tabla 3 ).

La -Tabla 4 describe que en las cuatro características comparadas no existen diferencias estadísticamente significativas con respecto al decremento porcentual de cada apartado del cuestionario, a excepción del tamaño y número de siembras en relación al apartado: "en cuanto a la enfermedad." Tampoco evidenciamos diferencias en el apartado "global." Lo que sí apreciamos es que, en todos

Tabla 2 Resultados descriptivos del cuestionario CAVICAVENMI a lo largo del seguimiento de los pacientes sin tratamiento adyuvante $(n=143)$

\begin{tabular}{|l|l|l|l|l|l|l|l|}
\hline & $\begin{array}{l}\text { Antes de } \\
\text { intervención } \\
\text { (media } \pm \text { DE) }\end{array}$ & $\begin{array}{l}\text { 1a visita }^{\text {post-intervención }} \\
\text { (media } \pm \text { DE) }\end{array}$ & $\mathrm{P}^{1}$ & $\begin{array}{l}\text { 2a visita }^{\text {post-intervención }} \\
\text { (media } \pm \text { DE) }\end{array}$ & $\mathrm{P}^{2}$ & $\begin{array}{l}\text { 3a visita }^{\text {post-intervención }} \\
\text { (media } \pm \text { DE) }\end{array}$ & $\mathrm{P}^{3}$ \\
\hline $\begin{array}{l}\text { En cuanto a la } \\
\text { enfermedad(a) }\end{array}$ & $8,2 \pm 3,4$ & $7,9 \pm 3,3$ & 0,253 & $7,2 \pm 3,6$ & 0,520 & $5,8 \pm 2,8$ & $<0,001$ \\
\hline $\begin{array}{l}\text { Autoestima y } \\
\text { estado } \\
\text { emocional(b) }\end{array}$ & $7,6 \pm 3,6$ & $6,9 \pm 3,5$ & 0,017 & $6,1 \pm 3,1$ & 0,010 & $4,1 \pm 2,2$ & $<0,001$ \\
\hline Vida laboral(c) & $1,1 \pm 1,6$ & $1,1 \pm 1,6$ & 0,946 & $0,7 \pm 1,3$ & $<0,001$ & $0,2 \pm 0,4$ & $<0,001$ \\
\hline Vida diaria(d) & $3,1 \pm 3,5$ & $2,8 \pm 3,2$ & 0,182 & $2,2 \pm 3,1$ & 0,010 & $0,7 \pm 1,3$ & $<0,001$ \\
\hline Vida sexual(e) & $3,4 \pm 2,7$ & $3,1 \pm 2,2$ & 0,791 & $2,7 \pm 2,6$ & 0,100 & $2,8 \pm 2,1$ & 0,016 \\
\hline Global(f) & $23,2 \pm 9,8$ & $22,8 \pm 9,5$ & 0,082 & $19,8 \pm 9,6$ & 0,402 & $13,5 \pm 5,7$ & $<0,001$ \\
\hline
\end{tabular}

$\mathbf{P}^{1}$ : Relación entre el momento antes de la intervención y la primera visita postintervención. $\mathbf{P}^{2}$ : Relación entre el momento antes de la intervención y la segunda visita postintervención. $\mathrm{P}^{3}$ : Relación entre el momento antes de la intervención y la tercera visita postintervención. (a): puntuación mínima 0, máxima 20; (b): puntuación mínima 0, má-xima 20 (c): puntuación mínima 0, máxima 8; (d): puntuación mínima 0, máxima 20; (e): puntuación mínima 0, máxima 16; (f): puntuación mínima 0, máxima 84 . 


\begin{tabular}{|c|c|c|c|c|c|c|c|}
\hline$\stackrel{\infty}{a}$ & $\begin{array}{l}\overline{8} \\
\circ \\
\circ\end{array}$ & $\begin{array}{l}\overline{8} \\
\overline{0} \\
\text { v }\end{array}$ & $\begin{array}{l}\overline{8} \\
0 \\
0\end{array}$ & $\begin{array}{l}\overline{8} \\
8 \\
0 \\
v\end{array}$ & $\left|\begin{array}{l}\tilde{O} \\
0 \\
0\end{array}\right|$ & & $\begin{array}{l}\overline{8} \\
0 \\
0 \\
V\end{array}$ \\
\hline & $\begin{array}{l}\dot{\sigma} \\
m \\
+1 \\
0 \\
\dot{n}\end{array}$ & $\begin{array}{l}6 \\
0 \\
+1 \\
+1 \\
- \\
-\end{array}$ & $\begin{array}{l}\infty \\
- \\
- \\
+ \\
- \\
-\end{array}$ & $\begin{array}{l}m \\
m \\
+1 \\
6 \\
\sim\end{array}$ & $\begin{array}{c}m \\
\tilde{N} \\
H \\
0 \\
m \\
m\end{array} \mid$ & & $\begin{array}{l}9 \\
\infty \\
+1 \\
0 \\
0 \\
0\end{array}$ \\
\hline à & $\begin{array}{l}\bar{J} \\
0 \\
0\end{array}$ & $\begin{array}{l}\overline{8} \\
\overline{0} \\
0 \\
\vee\end{array}$ & $\begin{array}{l}\bar{\sigma} \\
0 \\
V\end{array}$ & $\begin{array}{l}\infty \\
0 \\
0 \\
0 \\
V\end{array}$ & \begin{tabular}{c}
$n$ \\
\multirow{f}{0}{} \\
0 \\
$0^{-}$
\end{tabular} & & $\begin{array}{l}\overline{8} \\
0 \\
0 \\
V\end{array}$ \\
\hline 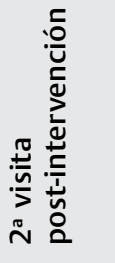 & $\begin{array}{l}n \\
m \\
+1 \\
\text { n } \\
\sigma^{-}\end{array}$ & $\begin{array}{c}0 \\
m \\
+1 \\
0 \\
6\end{array}$ & $\begin{array}{l}\infty \\
- \\
- \\
+ \\
\sim \\
-\end{array}$ & $\begin{array}{l}m \\
m \\
H \\
\infty \\
\infty \\
m\end{array}$ & $\begin{array}{c}\sigma \\
\tilde{N} \\
H \\
0 \\
\tilde{m} \\
\tilde{m}\end{array}$ & & $\begin{array}{l}\text { ñ } \\
\text { के } \\
+1 \\
\dot{0} \\
\dot{n}\end{array}$ \\
\hline$\stackrel{0}{0}$ & $\begin{array}{l}m \\
\stackrel{o}{q} \\
o \\
0\end{array}$ & $\begin{array}{l}0 \\
\stackrel{2}{N} \\
0\end{array}$ & $\begin{array}{l}\text { 등 } \\
\text { o }\end{array}$ & \begin{tabular}{l}
$m$ \\
\multirow{O}{0}{} \\
$0^{-}$
\end{tabular} & $\left|\begin{array}{l}0 \\
0 \\
0\end{array}\right|$ & & $\begin{array}{l}\overline{8} \\
0 \\
0 \\
v\end{array}$ \\
\hline 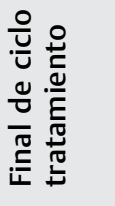 & $\begin{array}{c}0 \\
m \\
H \\
\sim \\
\tilde{\sigma}\end{array}$ & $\begin{array}{l}\infty \\
m \\
+1 \\
0 \\
\sigma\end{array}$ & $\begin{array}{l}\tilde{z} \\
\bar{H} \\
\tilde{n} \\
\tilde{n}\end{array}$ & $\begin{array}{l}- \\
\sigma^{-} \\
+ \\
m \\
\sigma^{-}\end{array}$ & $\begin{array}{c}\tilde{L} \\
\sim \\
H \\
0 \\
0 \\
f\end{array}$ & $\mid \begin{array}{c}\infty \\
m \\
+1 \\
r \\
0^{-} \\
-\end{array}$ & $\begin{array}{l}0 \\
m \\
\dot{n} \\
+1 \\
0 \\
\dot{q}\end{array}$ \\
\hline$\stackrel{\text { nq }}{a}$ & $\frac{0}{\check{\sigma}}$ & $\begin{array}{l}\text { बे } \\
\text { ஸ̂ } \\
0\end{array}$ & $\begin{array}{l}\text { 오 } \\
\text { O } \\
0\end{array}$ & $\begin{array}{l}0 \\
0 \\
0 \\
0\end{array}$ & $\left|\begin{array}{l}m \\
\tilde{m} \\
0 \\
0\end{array}\right|$ & & $\begin{array}{l}\overline{8} \\
0 \\
0 \\
V\end{array}$ \\
\hline 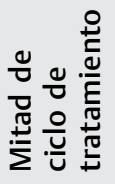 & $\begin{array}{l}\hat{\tilde{m}} \\
+ \\
\tilde{H} \\
\tilde{0}\end{array}$ & $\begin{array}{l}0 \\
\dot{m} \\
+1 \\
\bar{\sigma} \\
\sigma\end{array}$ & $\begin{array}{l}\bar{i} \\
H \\
m \\
m\end{array}$ & $\begin{array}{c}N \\
\forall \\
H \\
m \\
r\end{array}$ & $\begin{array}{c}0 \\
\tilde{N} \\
H \\
0 \\
0 \\
\forall\end{array}$ & $\begin{array}{c}\tilde{y} \\
m \\
+ \\
\infty \\
\infty \\
0 \\
-\end{array}$ & $\begin{array}{l}\hat{n} \\
\tilde{n} \\
H \\
\tilde{f} \\
\dot{f}\end{array}$ \\
\hline 茄 & $\begin{array}{l}\bar{j} \\
\text { m. } \\
0\end{array}$ & 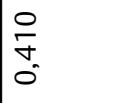 & $\begin{array}{l}\hat{\alpha} \\
\text { กิ } \\
\text { o }\end{array}$ & $\begin{array}{l}\frac{N}{6} \\
0 \\
0\end{array}$ & $\mid \begin{array}{l}0 \\
m \\
\infty \\
0 \\
0\end{array}$ & $\begin{array}{l}0 \\
+ \\
5 \\
0 \\
0\end{array}$ & $\begin{array}{c}\bar{f} \\
\text { m. } \\
0\end{array}$ \\
\hline 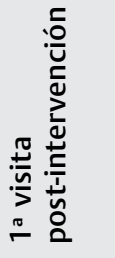 & $\begin{array}{l}0 \\
\dot{+} \\
H \\
\infty \\
\infty\end{array}$ & $\begin{array}{l}n \\
\tilde{m} \\
+1 \\
0 \\
\infty\end{array}$ & $\begin{array}{l}10 \\
\sim \\
H \\
0 \\
0 \\
-\end{array}$ & $\begin{array}{c}0 \\
\sigma \\
+1 \\
\tilde{m} \\
\tilde{m}\end{array}$ & $\begin{array}{l}0 \\
\sim \\
+1 \\
\sigma \\
\sim \\
\sim\end{array} \mid$ & & $\begin{array}{l}0 \\
m \\
\dot{n} \\
+ \\
\tilde{d}\end{array}$ \\
\hline 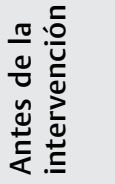 & $\begin{array}{l}\hat{m} \\
\dot{m} \\
m \\
\infty\end{array}$ & $\begin{array}{l}0 \\
m \\
+1 \\
r \\
\infty\end{array}$ & $\begin{array}{l}q \\
- \\
+1 \\
m \\
n\end{array}$ & $\begin{array}{c}\sigma \\
m \\
H \\
\sim \\
\sim \\
\forall\end{array}$ & $\begin{array}{l}\tilde{N} \\
\tilde{N} \\
\tilde{\sigma} \\
\tilde{N}\end{array}$ & & $\begin{array}{l}0 \\
\infty \\
+1 \\
m \\
m \\
\stackrel{n}{N}\end{array}$ \\
\hline & 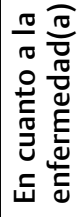 & 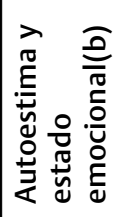 & 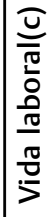 & 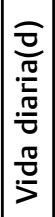 & 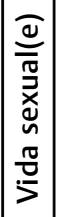 & 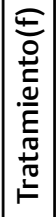 & 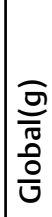 \\
\hline
\end{tabular}

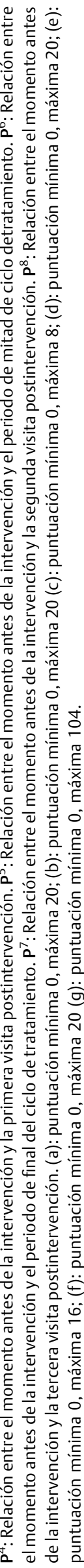

Tabla 4 Características tumorales y calidad de vida en la tercera visita postintervención

\begin{tabular}{|l|l|l|l|}
\hline & $\begin{array}{l}\text { Calidad de vida } \\
\text { (mean } \pm \text { DE) }\end{array}$ & p & p ajustada \\
\hline Tamaño (cm) & $19,9 \pm 10,6$ & 0,081 & 0,151 \\
\hline$\geq 3$ & $18,1 \pm 8,6$ & & \\
\hline$<3$ & $18,4 \pm 10,3$ & 0,218 & - \\
\hline Siembras & $18,7 \pm 8,9$ & & \\
\hline$\geq 3$ & & & \\
\hline$<3$ & $21,1 \pm 10,3$ & 0,077 & 0,105 \\
\hline Localización & $19,8 \pm 8,7$ & & \\
\hline Trígono y/o cúpula & & & \\
\hline Otra & & 0,791 & - \\
\hline Carcinoma in situ & $18,6 \pm 9,7$ & & \\
\hline Presencia & $18,3 \pm 9,5$ & & \\
\hline Ausencia &
\end{tabular}

los casos, las puntuaciones obtenidas son mayores en presencia de las características tumorales.

En el estudio univariante, dos de las cuatro características tumorales: tamaño $\geq 3 \mathrm{~cm}$ y localización del tumor, están próximas a explicar su influencia de manera independiente en la calidad de vida: $p=0.081$ y $p=0.077$ respectivamente. Pero cuando se realiza el estudio de regresión logísitica, ninguna de ellas explica de manera independiente su influencia en dicha calidad de vida, resultando $\mathrm{p}>0.05$ en todos los casos (- Tabla 5).

\section{Discusión}

En este estudio se ha evaluado la calidad de vida en pacientes con TVNMI utilizando el cuestionario CAVICAVENMI.

Tanto en la Tabla 2 como en la 3 entre el momento antes de la cirugía y primera visita postintervención, las diferencias encontradas en la calidad de vida son mínimas, pero a medida que transcurre el tiempo, la evolución de las respuestas presenta una mejora en la mayoría de los apartados. Eso puede ser debido a que cuando en los controles evolutivos los pacientes se ven libres de enfermedad y no han presentado recidiva de la hematuria, se encuentran anímicamente mejor, lo que repercute positivamente en el cuestionario. La dimensión más influenciada desde un principio y que antes responde al cambio, es la autoestima y el estado emocional, puesto que la mayoría de los pacientes, antes de la intervención responden a las preguntas 4 y 5(anexo 1) de este apartado, con una puntuación máxima, y a medida que ven cómo evoluciona su enfermedad esa puntuación cae. Esos resultados coinciden con el estudio de $\mathrm{Schmidt}^{9}$ quien realiza un estudio prospectivo observacional de calidad de vida en pacientes con TVNMI donde plasma esos resultados en el apartado patients mental health. En lo referente al apartado "en cuanto a la enfermedad" muchos pacientes, sobre todo aquellos con presencia de las características tumorales previamente descritas, presentan sintomatología irritativa a 
Tabla 5 Estudio de asociación entre el decremento porcentual (situación basaltercera visita postintervención) y las características tumorales $(n=180)$

\begin{tabular}{|c|c|c|c|c|c|c|}
\hline & $\begin{array}{l}\text { En cuanto a la } \\
\text { enfermedad } \\
\text { (media } \pm \text { DS) }\end{array}$ & $\begin{array}{l}\text { Autoestima y } \\
\text { estado emocional } \\
\text { (media } \pm \text { DS) }\end{array}$ & $\begin{array}{l}\text { Vida laboral } \\
\text { (media } \pm \text { DS) }\end{array}$ & $\begin{array}{l}\text { Vida diaria } \\
\text { (media } \pm \text { DS) }\end{array}$ & $\begin{array}{l}\text { Vida sexual } \\
\text { (media } \pm \text { DS) }\end{array}$ & $\begin{array}{l}\text { Global } \\
(\text { media } \pm \text { DS) }\end{array}$ \\
\hline \multicolumn{7}{|c|}{ Tamaño $(\mathrm{cm})$} \\
\hline$\geq 3$ & $35,7 \pm 28,2$ & $44,6 \pm 32,1$ & $36,4 \pm 25,3$ & $61,3 \pm 31,1$ & $20,5 \pm 31,9$ & $41,8 \pm 22,7$ \\
\hline$<3$ & $21,0 \pm 21,6$ & $43,2 \pm 26,0$ & $27,4 \pm 20,2$ & $50,4 \pm 35,4$ & $19,8 \pm 34,7$ & $33,11 \pm 20,4$ \\
\hline$P$ & 0,013 & 0,700 & 0,400 & 0,080 & 0,800 & 0,130 \\
\hline \multicolumn{7}{|l|}{ Siembras } \\
\hline$\geq 3$ & $37,3 \pm 26,1$ & $44,9 \pm 30,4$ & $33,3 \pm 35,3$ & $58,8 \pm 42,5$ & $20,9 \pm 32,7$ & $40,3 \pm 23,4$ \\
\hline$<3$ & $19,7 \pm 22,7$ & $40,2 \pm 32,6$ & $29,3 \pm 32,4$ & $52,5 \pm 42,8$ & $18,0 \pm 34,0$ & $34,4 \pm 20,8$ \\
\hline$P$ & 0,050 & 0,600 & 0,700 & 0,300 & 0,700 & 0,100 \\
\hline \multicolumn{7}{|l|}{ Localización } \\
\hline Trígono y/o & $30,8 \pm 25,7$ & $46,6 \pm 25,8$ & $33,5 \pm 28,2$ & $59,4 \pm 38,8$ & $23,9 \pm 34,7$ & $40,2 \pm 20,3$ \\
\hline \multicolumn{7}{|l|}{ Cúpula } \\
\hline Otra & $25,6 \pm 23,7$ & $42,0 \pm 30,7$ & $30,1 \pm 27,1$ & $52,6 \pm 45,1$ & $17,4 \pm 32,2$ & $34,9 \pm 23,3$ \\
\hline$P$ & 0,200 & 0,300 & 0,800 & 0,300 & 0,200 & 0,100 \\
\hline \multicolumn{7}{|c|}{ Carcinoma in situ } \\
\hline Presencia & $34,1 \pm 25,6$ & $49,7 \pm 24,7$ & $52,8 \pm 36,1$ & $54,0 \pm 40,0$ & $15,9 \pm 30,1$ & $46,8 \pm 19,7$ \\
\hline Ausencia & $26,7 \pm 25,8$ & $43,0 \pm 29,7$ & $48,1 \pm 31,6$ & $55,6 \pm 43,2$ & $20,7 \pm 33,7$ & $35,6 \pm 22,5$ \\
\hline$P$ & 0,200 & 0,300 & 0,200 & 0,800 & 0,500 & 0,090 \\
\hline
\end{tabular}

causa del tumor, que provoca unas puntuaciones más elevadas al inicio del estudio y que van decreciendo a medida que pasan el periodo convalenciente postquirúrgico y se ha eliminado la causa de las molestias urinarias. Schmidt y col. ${ }^{9}$ describen que más de $2 / 3$ de sus pacientes estudiados mejoran la sintomatología miccional tras la intervención. En cuanto a la vida diaria, que también se modifica en gran medida, relacionamos el cambio en ese parámetro con que el paciente una vez operado se aleja de la fecha de intervención incorporándose a su vida diaria habitual y reestableciendo su día a día. En el apartado de vida laboral, las puntuaciones desde el principio son bastante bajas, es decir que los pacientes mantienen una calidad de vida relativamente buena pese a la enfermedad; eso podemos achacarlo a que la edad media de nuestros pacientes es de 70,3 años, por lo que la mayoría son jubilados y la mayor parte varones y su vida laboral o las tareas que desempeñan en su domicilio se ven poco afectadas. En cuanto a la esfera sexual, los artículos citados anteriormente ${ }^{5,7,9}$ junto con el nuestro, apenas perciben cambios en la escala sexual, puede ser porque ni la patología ni las intervenciones realizadas repercuten físicamente en la misma y es un aspecto que se mueve en un rango de puntuaciones bajas probablemente por la edad media de los pacientes.

El subgrupo de pacientes que reciben BCG o MMC parten de un estado de calidad de vida similar al resto de pacientes, pero, aunque su calidad de vida se ve más afectada cuando están recibiendo el ciclo de tratamiento, la mejora en la calidad de vida es más prematura que en el grupo de pacientes que no reciben instilaciones, observando unas diferencias significativas en todos los apartados en la segunda visita postintervención. Creemos que, debido a la gran afectación que les produce el tratamiento sobre el estado general cuando terminan con las instilaciones, mejora radicalmente su calidad de vida. Uno de los apartados que más se modifica es el denominado "en cuanto a la enfermedad", incluso reduciendo la puntuación en 5 puntos, eso mismo lo reflejan Smicht ${ }^{9}$ como mejoría de los urinary problems y Blazeby ${ }^{5}$ que lo cita como urinary and future worries. Ninguno de los estudios hace mención a la vida diaria, laboral ni a la dimensión del cuestionario denominada tratamiento. Pero pensamos que hay que hacer una mención a ello ya que durante el ciclo de tratamiento endovesical, observamos un empeoramiento en la calidad que repercute de manera importante en el día a día de los pacientes obligándoles a modificar sus actividades diarias, lo cual incide negativamente en su estado de autoestima y estado emocional, vida familiar y sexual. Por lo tanto, creemos que los pacientes tienen que ser bien informados sobre la necesidad del tratamiento adyuvante y el deterioro en la calidad de vida que ello conlleva.

La comparación de los decrementos porcentuales, aunque no han obtenido diferencias estadísticamente significativas, plasman una peor calidad de vida en presencia de las características tumorales. Así, los pacientes que presentan al menos una de las características tumorales, tienen una clínica irritatia miccional más acusada, lo que provoca imperiosidad a la ora de orinar y escapes de orina. Como hemos descrito en la - Tabla $\mathbf{4}$, esos pacientes presentan 
mayor puntuación en el cuestionario, lo que se traduce en peor calidad de vida.

También pretendemos valorar si la presencia de dichas características tumorales influye de manera independiente en la calidad de vida de pacientes TVNMI, pero en el estudio multivariante queda demostrado que ninguna de ellas tiene influencia de manera significativa.

Una de las limitaciones de este estudio es que no podemos comparar los resultados con lo publicado en la literatura, porque como hemos indicado anteriormente, los grupos de comparación no presentan las mismas características.

Sería razonable que continuáramos este estudio a lo largo del tiempo para ver la evolución de la calidad de vida en esos pacientes cuando presentan remisión completa y cómo responden los pacientes que presentan recidiva tumoral. También encontramos útil que este cuestionario se adaptara a otras lenguas y culturas para poder protocolizar la información administrada al paciente diagnosticado de TVNMI y así resolver las dudas surgidas y los procesos intercurrentes con el fin de mejorar en todo lo posible su calidad de vida y que ellos puedan afrontar la enfermedad con una mejor perspectiva. Dado que, con los resultados obtenidos, observamos que al inicio del estudio área de autoestima y estado emocional se altera en gran medida y que posteriormente, a medida que pasa el tiempo esa puntuación mejora, debemos pensar que los pacientes están desinformados en cuanto a las características de la patología, su alta tasa de recidiva y no muy alta mortalidad.

\section{Conclusiones}

La calidad de vida en pacientes TVNMI, presenta una mejoría a lo largo de la evolución del estudio y que es mayor cuanto más tiempo haya pasado desde la intervención. La calidad de vida en pacientes TVNMI presenta una mejoría a lo largo de la evolución del estudio. Evidenciamos un cambio más temprano en la calidad de vida en los pacientes que reciben tratamiento adyuvante. Aquellos pacientes con presencia de las características tumorales descritas (tamaño $>3 \mathrm{~cm}$, localización en cúpula y trígono, numero de siembras $>3$ y CIS positivo), presentan un estado de peor calidad de vida. Los apartados en donde se encuentra un cambio más acusado de la calidad de vida son: "en cuanto a la enfermedad" y "autoestima y estado emocional." Los pacientes están desinformados en lo que se refiere a la alta tasa de recidiva y no muy alta mortalidad de la patología que padecen. Por todo eso, concluimos que el médico debería ofrecer una información más detallada a los pacientes diagnosticados con esa patología, en cuanto a su fisiopatología, tratamiento, pronóstico y evolución.

\section{Responsabilidades Éticas}

Protección de personas y animales. Los autores declaran que para esta investigación no se han realizado experimentos en seres humanos ni en animales.

Confidencialidad de los datos. Los autores declaran que han seguido los protocolos de su centro de trabajo sobre la publicación de datos de pacientes.

Derecho a la privacidad y consentimiento informado. Los autores declaran que en este artículo no aparecen datos de pacientes.

\section{Conflicto de Intereses}

No exsiste conflicto de intereses por parte de ninguno de los autores.

\section{Bibliografía}

1 WHOQOL Group. Study protocol for the World Health Organization project to develop a Quality of Life assessment instrument (WHOQOL). Qual Life Res 1993;2(02):153-159

2 The World Health Organization Quality of Life assessment (WHOQOL): position paper from the World Health Organization. Soc Sci Med 1995;41(10):1403-1409

3 Testa MA, Simonson DC. Assessment of quality-of-life outcomes. N Engl J Med 1996;334(13):835-840

4 Babjuk M, Oosterlinck W, Sylvester R, et al; Asociación Europea de Urología. Guía clínica del carcinoma urotelial de vejiga no músculo-invasivo de la Asociación Europea de Urología. Actualización de 2011. Actas Urol Esp 2012;36(07):389-402

5 Blazeby JM, Hall E, Aaronson NK, et al. Validation and reliability testing of the EORTC QLQ-NMIBC24 questionnaire module to assess patient-reported outcomes in non-muscle-invasive bladder cancer. Eur Urol 2014;66(06):1148-1156

6 Schmidt S, Riel R, Frances A, et al; EMPARO-CU Study Group. Bladder cancer index: cross-cultural adaptation into Spanish and psychometric evaluation. Health Qual Life Outcomes 2014;12:20

7 Gilbert SM, Dunn RL, Hollenbeck BK, et al. Development and validation of the Bladder Cancer Index: a comprehensive, disease specific measure of health related quality of life in patients with localized bladder cancer. J Urol 2010;183(05):1764-1769

8 Abáigar-Pedraza I, Megías-Garrigós J, Sánchez-Payá J. Cuestionario de calidad de vida para pacientes con cáncer de vejiga no músculo invasivo. Actas Urol Esp 2016;40(04):251-257

9 Schmidt S, Francés A, Lorente Garin JA, et al. Quality of life in patients with non-muscle-invasive bladder cancer: one-year results of a multicentre prospective cohort study. Urol Oncol 2015;33(01):19.e7-19.e15 